\title{
国留
}

\section{Improved vascularity in a scarred post burn transposition flap}

Wan Loong James Mok MRCS(Ed), Si Jack Chong MRCS(Ed) FAMS, Rei Ogawa MD PhD FACS

\section{Background / Aim}

Massive burn injury often has scar complications persisting long after the patient has survived the acute phase. These include neck and limb contractures, eyelid ectropion, microstomia, hypertrophic and keloid scarring, itch and dysesthesia. We present a case of $46 \%$ total body surface area burn with recurrent left neck contracture in which a large perforator based transposition flap was performed. Our aim was to compare the vascularity of scarred, post burn skin flaps versus traditional local flaps.

\section{Methods}

A 20x5cm transposition flap was planned over the right chest. Preoperatively, a Doppler probe was used to identify thoracoacromial perforators at the flap axis and internal mammary perforators supplying the distal flap. The fasciocutaneous flap was raised, preserving two distal perforators. Full thickness excision of the left neck contracture was performed and the flap was transposed superiolaterally to fit the defect.

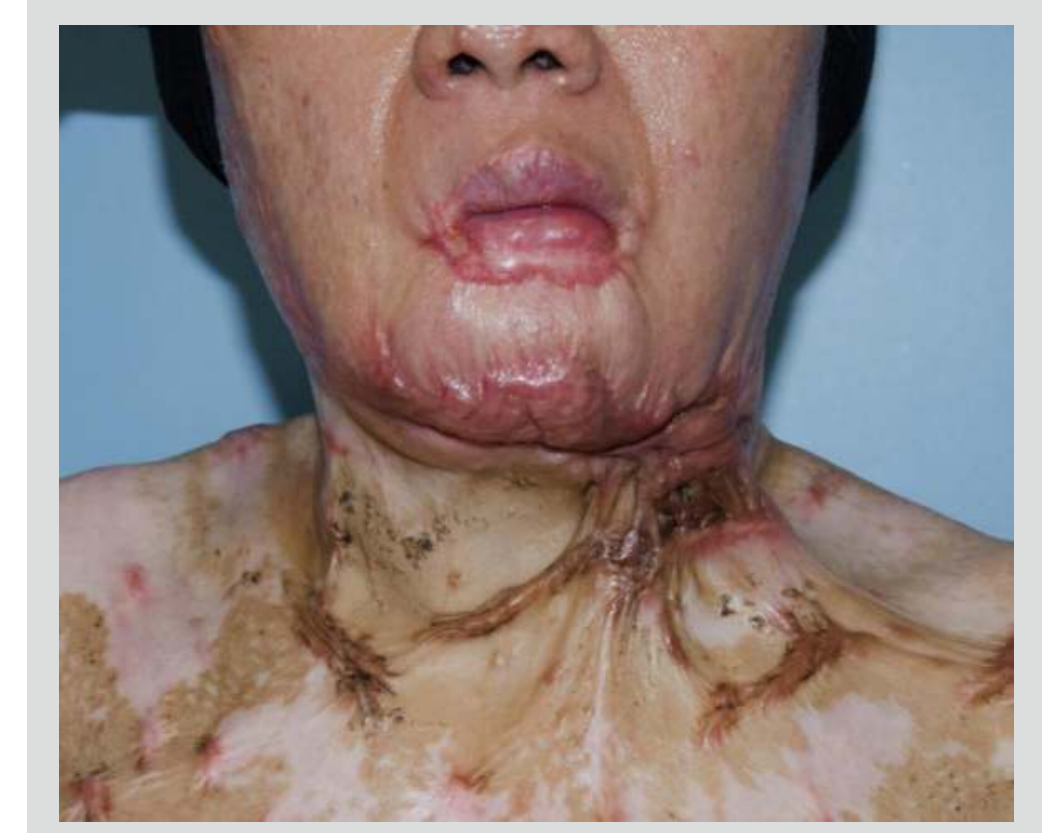

\section{Left neck contracture}

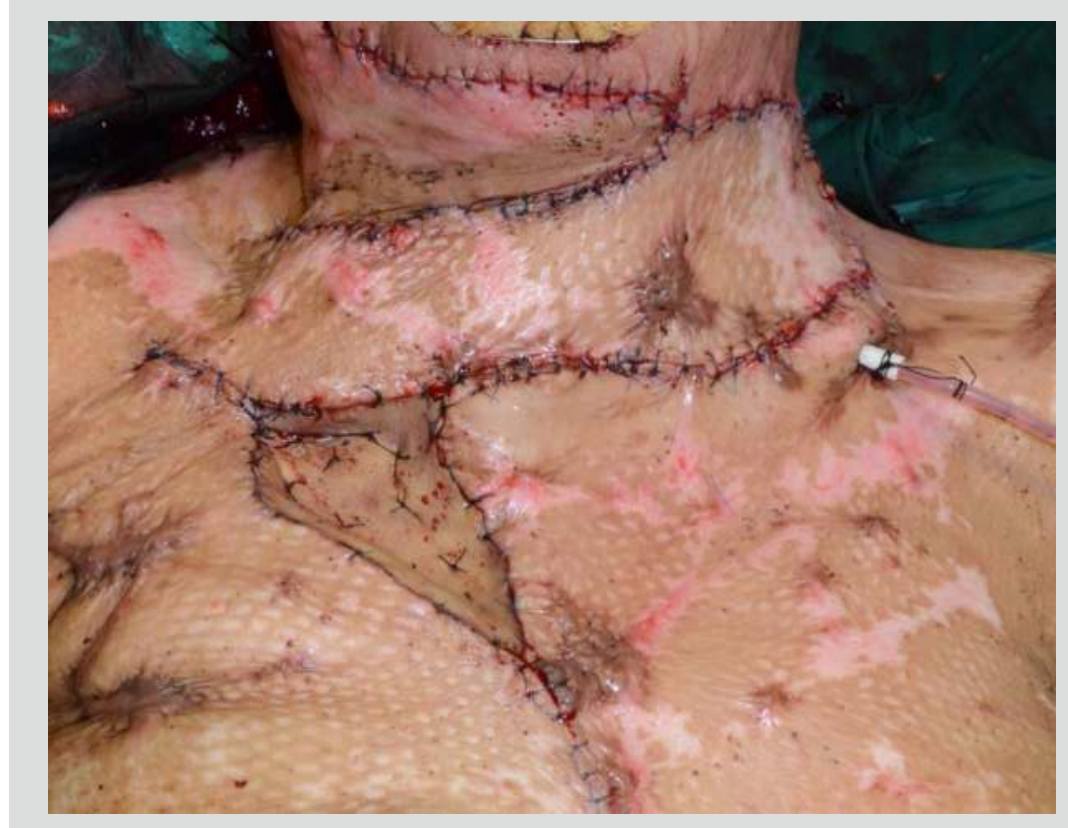

Flap appearance after operation

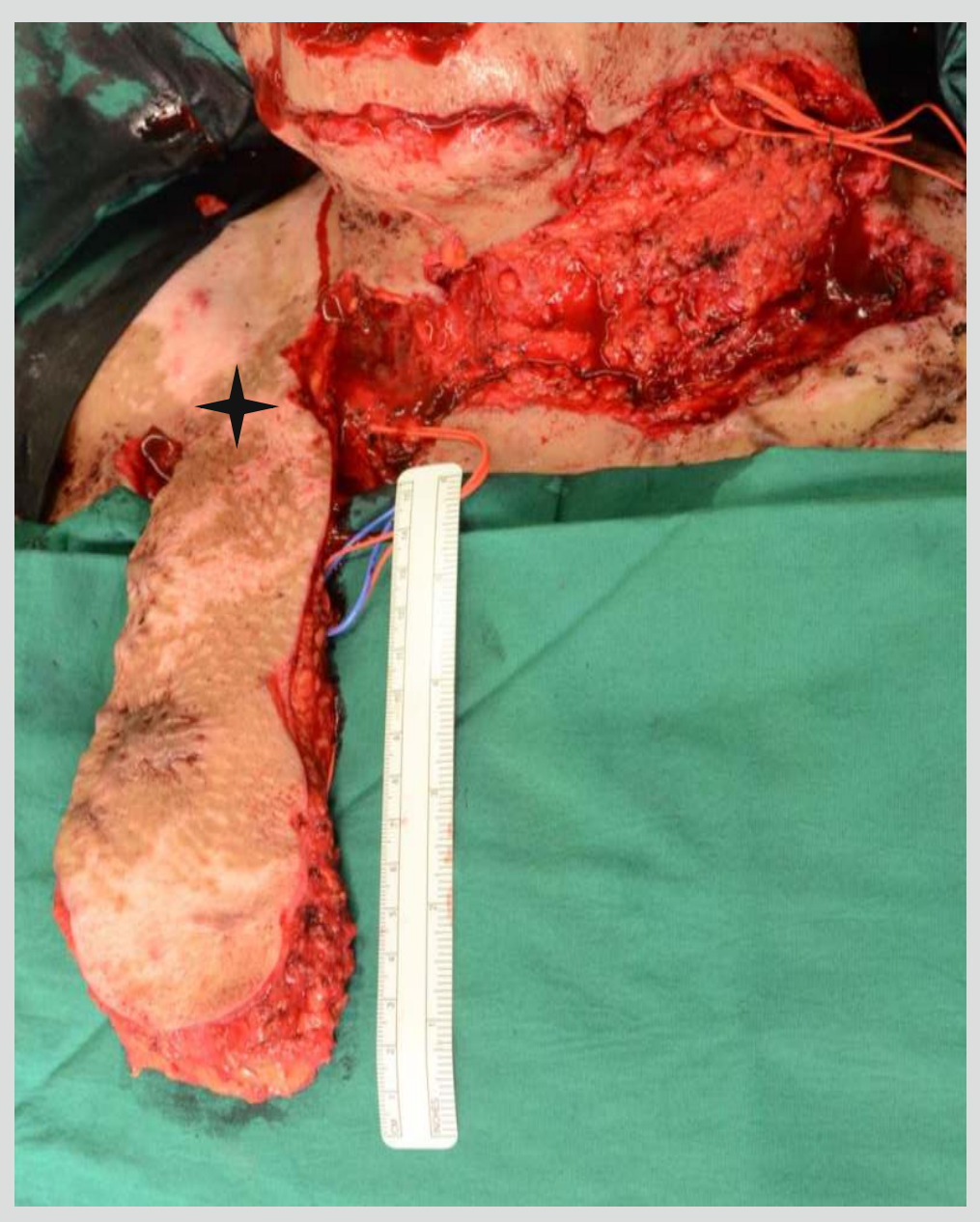

\section{Thoracoacromial artery perforator flap raised; good vascularity over distal tip of flap $\left(^{*}\right)$ Flap axis}

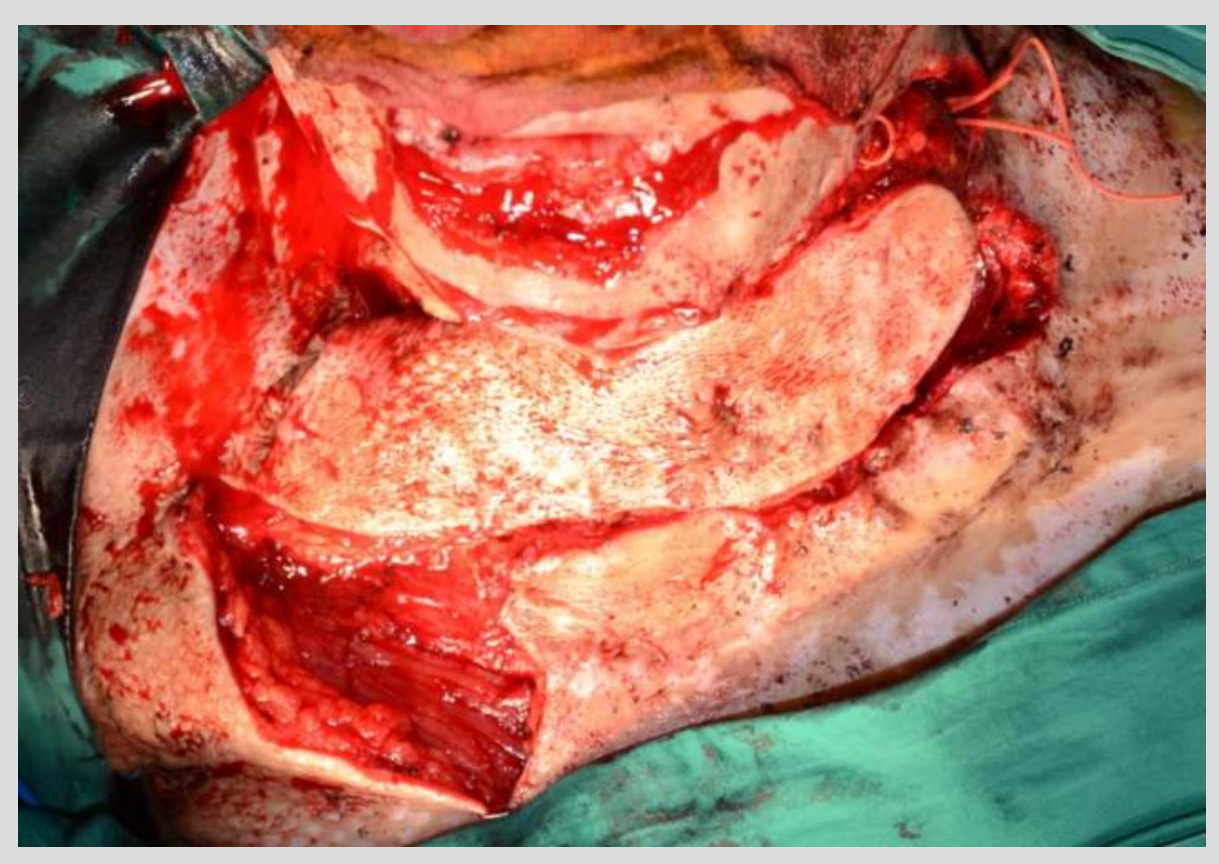

Flap transposition

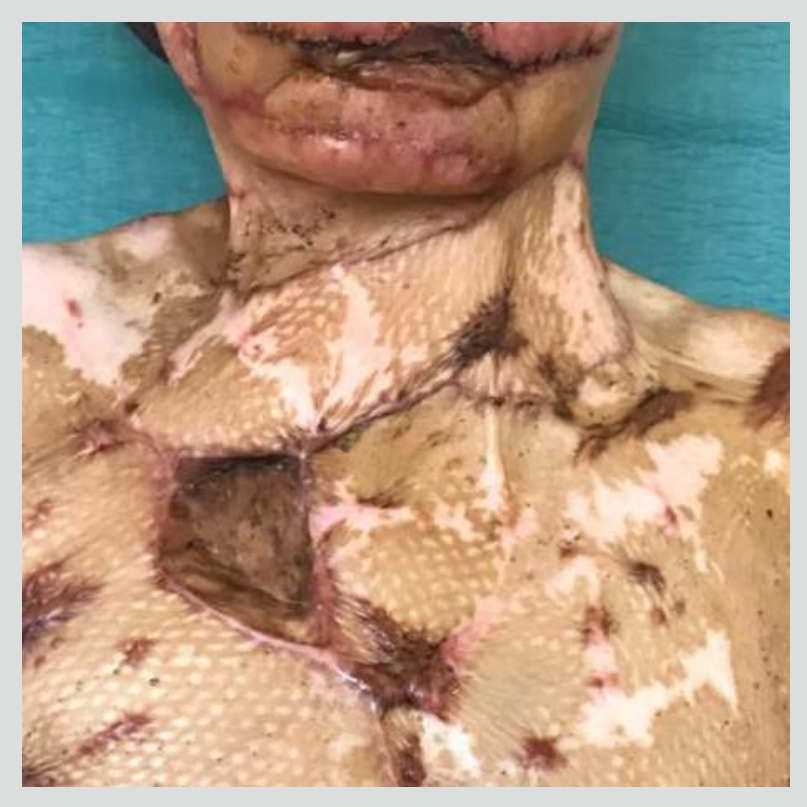

2 months post op. No flap necrosis

\section{Results / Conclusion}

Flap vascularity at its distal edges was excellent despite the length of flap. Microvascular augmentation of distal arterial flow was not required. The flap remained well perfused with no necrosis.

Large transposition flaps with narrow bases tend to suffer from distal flap necrosis. This can usually be addressed at the outset by preserving distal flap perforators for vascular augmentation at the recipient site. In our case, we noticed improved vascularity over scarred post burn tissue hence there was no need for vascular augmentation. Unusual lengths of flap can be taken due to the delay phenomenon of burn injury. 